\title{
Active haptic detection and discrimination of shape
}

\author{
S. LOUW, A. M. L. KAPPERS, and J. J. KOENDERINK \\ Utrecht University, Utrecht, The Netherlands
}

\begin{abstract}
In previous research, we have shown that detection thresholds for Gaussian shapes increase with a power of 1.3 of spatial width. In the present three experiments, we generalized this finding to more complex shapes and to discrimination tasks. In Experiment 1, we found that the slope of the psychometric function for detection (i.e., distinguishing curved from flat surfaces) was independent of surface shape. In Experiment 3, we found the same result for discrimination of two different curved shapes. In Experiment 2, we found that detection and discrimination functions had the same dependence on spatial width, except that discrimination thresholds were two to four times larger. Possible neural mechanisms underlying these results are discussed.
\end{abstract}

Human haptic perception of physical shape is mediated by a wide variety of mechanisms. A physical stimulus deforms the skin, and different kinds of mechanoreceptors detect these deformations. Next, afferent nerves transfer the resulting signals to the brain. Tactile units encode these signals. In contrast to the vast amount of knowledge about the constituent parts of haptic perception, the combination and integration of these parts are poorly understood.

In the study reported here, we focused on the assessment of haptic detection and discrimination thresholds of the shape of objects. Here, the detection task was to distinguish curved from flat surfaces. Discrimination is judging which one of two curved surfaces has a specific shape. Haptic exploration-the scanning of objects by active movements of fingers, arms, and shoulders - is of high importance for our acquisition of information about the environment. Detection and discrimination thresholds define the limits of the haptic system. Knowledge of these limits enables us, for example, to optimize the presentation of real and virtual haptic information. Thresholds for normal haptic perception may also serve as a benchmark to pinpoint malfunctioning haptic systems.

Studies were selected in which the methods and materials used corresponded to the experiments described here. Quantitative data on detection and discrimination thresholds of human haptic exploration of well-defined objects with the fingertips turned out to be scarce. We found five studies that yielded data with which our experiments can be compared (Davidson, 1972; Goodwin, John, \& Marceglia, 1991; Gordon \& Morison, 1982; Louw, Kappers, \& Koenderink, 2000; Pont, Kappers, \& Koenderink, 1999). In all these studies, detection thresholds were measured

This research was supported by the Netherlands Organization for Scientific Research (NWO). Correspondence should be addressed to A. M. L. Kappers, Utrecht University, Helmholtz Instituut, Princetonplein 5, 3584 CC Utrecht, The Netherlands (e-mail: a.m.l.kappers@ phys.uu.nl). for one or more widths of a stimulus. When all data from the experiments are taken together, the thresholds show a clear pattern (see Louw et al., 2000). In two of these studies-namely, those by Gordon and Morison (1982) and Pont et al. (1999) - the scanned length of circular cylindrically shaped stimuli was varied. In both studies, the authors concluded that the effective stimulus for haptic detection is the maximum slope difference over the surface. Because these conclusions were drawn on the basis of a limited range of the total range of spatial haptic perception, some uncertainty remains. In the most recent study (Louw et al., 2000), threshold amplitudes were measured using Gaussian shaped stimuli convex and concave Gaussian shapes are depicted in uper part of Figure 1. They concluded that, measured over the whole range of spatial haptic perception, detection threshold amplitudes increase with a power of 1.3 of the width of the Gaussian shape. In addition, both Goodwin et al. (1991) and Louw et al. (2000) provided strong evidence that detection thresholds for convex and concave stimuli do not differ significantly.

Our aims in the present study were twofold. First, we wanted to verify whether the power law dependence has a more general application than for zeroth-order Gaussians or circular cylindrical profiles alone. Second, we wanted to test whether the power law dependence of detection thresholds on spatial dimensions also yields a good description of discrimination thresholds. We approached these questions by measuring both detection thresholds of four different shapes and discrimination thresholds of six different combinations of the shapes.

The shapes we wanted to experiment with had to meet the following requirements: They had to be smooth over the whole surface, and their height and spatial width had to be able to be varied independently. Smoothness was required to have a well-defined slope and curvature over the whole length of the stimulus. Furthermore, we wanted the stimuli to have the same profiles, irrespective of the direction in which they were probed, so the profiles had to 
be symmetric. The Gaussian shape and its even-order derivatives meet these criteria. We picked the zeroth-order derivative and the second-order derivative, because they are the simplest even derivatives for our purposes. A further variation in shape was achieved by simply taking the convex and concave counterparts of both zeroth- and secondorder Gaussian derivatives (See Figure 1). Although somewhat similar at first glance, the four shapes are markedly different. The number and sequence of convex-concave transitions of all shapes is clearly distinct. Additionally, the zeroth- and second-order Gaussians differ in their proportions between maximum amplitude, slope, and curvature. The spatial width of the stimuli is indicated by $\sigma$. In statistics, $\sigma$ is known as the standard deviation of a Gaussian distribution. Two $\sigma \mathrm{s}, 7.5$ and $60 \mathrm{~mm}$, were chosen for each of the four shapes, as we also aimed to test for the dependence of thresholds on stimulus width.

Louw et al. (2000) measured detection thresholds of convex and concave Gaussian shapes with $\sigma$ in the range between $150 \mu \mathrm{m}$ and $240 \mathrm{~mm}$. The dimensions of stimuli in the present study lie well within this range and at the same time they lie far enough apart to obtain a good estimate of the power factor. In the present study, data were expanded in several ways. In Experiment 1, participants had to discriminate Gaussian shapes from a flat reference surface. Thus, detection thresholds for the four shapes were obtained. In Experiment 2, we measured both detection thresholds and thresholds for discrimination between shapes. The two thresholds were compared with each other. Experiment 3 concerned discrimination between the four different shapes. This provided us with thresholds for six difference shapes, as explained in the Analysis section of Experiment 3 . The dependence of these six thresholds on shape and spatial width was examined. We tried to determine whether the factors of the power law dependence between thresholds and spatial width in each experimental condition were in accordance with each other and with Louw et al. (2000). If the same factor of the power law is found in each condition, it must reflect a general property of haptic perception.

\section{EXPERIMENT 1}

In Experiment 1, we aimed to find the effective stimulus for the detection of the four different shapes. We wanted to know whether there is one specific property of the different shapes that describes all detection thresholds. Louw et al. (2000) established that thresholds depend on $\sigma$ with a power law over the whole range of spatial scales. In the present experiment, we tried to confirm whether the power law with factor 1.3 generalizes across more complex stimuli.
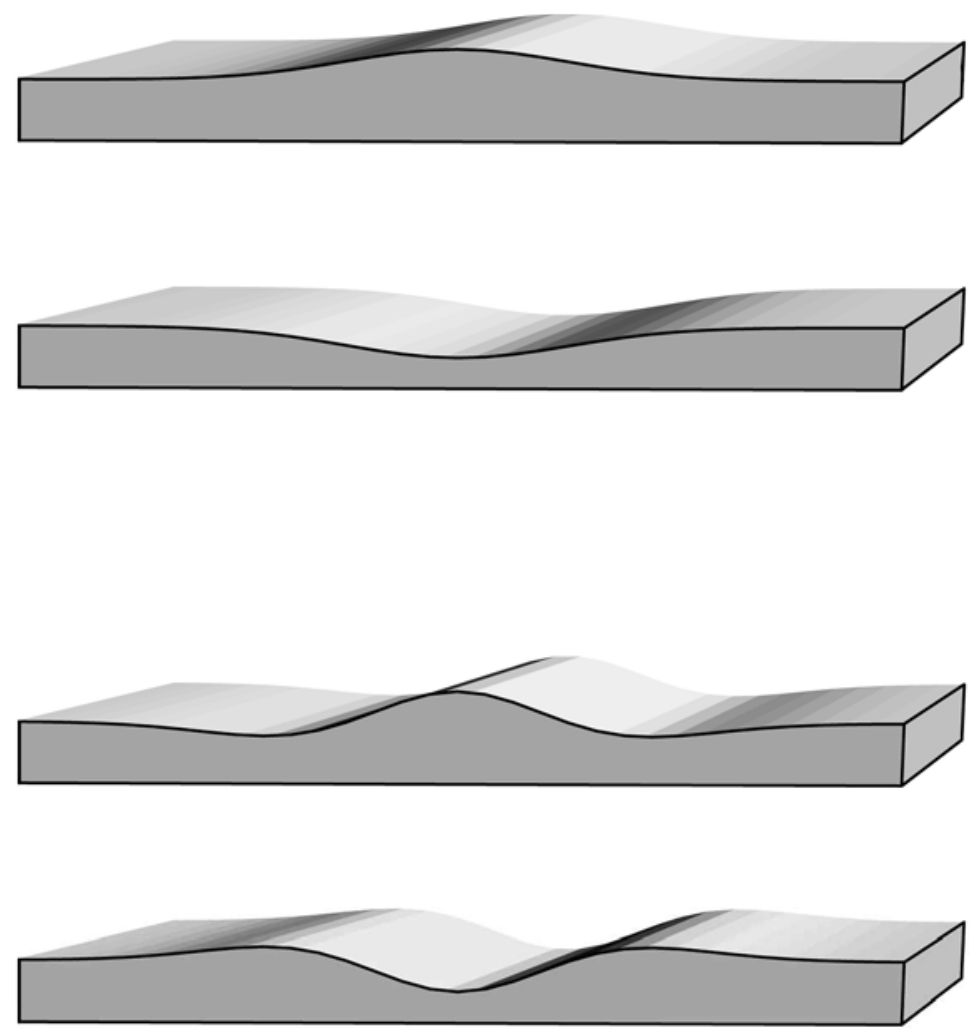

Figure 1. Schematic drawing of the stimuli: a convex and a concave Gaussian surface (above) and their second derivatives (below), defined as convex and concave, respectively. The amplitudes of the profiles are exaggerated dramatically. 


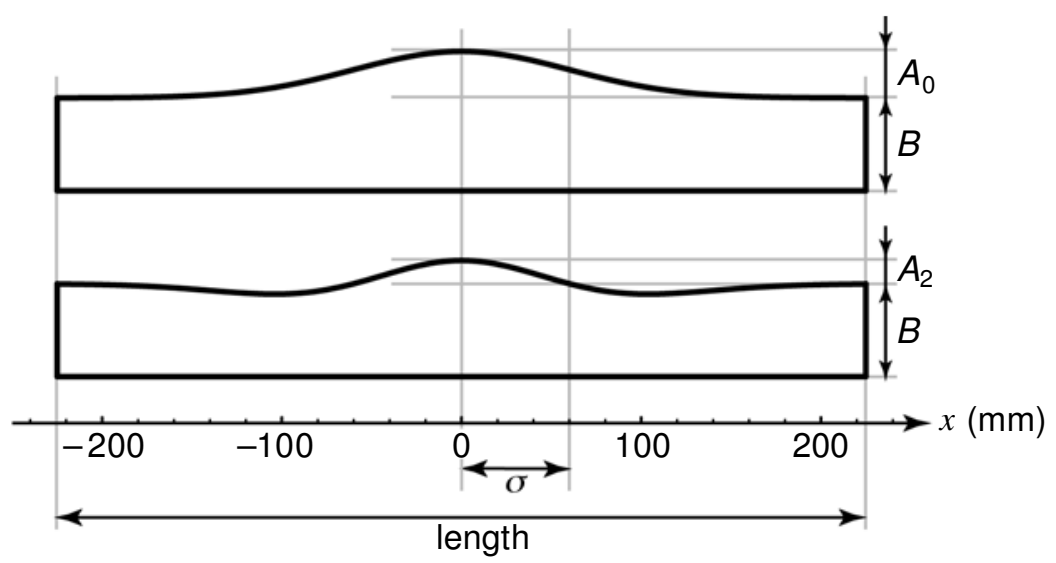

Figure 2. Schematic drawing of the stimuli: a Gaussian surface (above) and its second derivative (below). The dimensions of the strip, length, and base height $(B)$ are independent of the dimensions of the profiles, amplitude $(A)$, and $\sigma$.

\section{Method}

Participants. Four people participated in the experiment: $1 \mathrm{fe}-$ male student (I.S.) and 3 male students (B.H., J.S., and J.V.). The participants were ignorant of the purpose of the experiment, and they were paid for their efforts. According to Coren's (1993) test, 3 participants were strongly right-handed, and 1 (J.S.) was weakly lefthanded.

Stimuli. The stimuli consisted of strips, their upper surface having a curved profile. The symmetry line of the curve ran along the middle of the strip. When $x$ is taken as a coordinate in the length direction of the strip with the origin at the center, the height of the Gaussian surface, $h_{0}(x)$, is given by

$$
h_{0}(x)=B+A_{0} e^{-\frac{x^{2}}{2 \sigma^{2}}},
$$

where $\sigma$ is the spatial width of the surface (see Figure 2). The amplitude, $A_{0}$, of the surface is the difference between the most extreme height and the base height, $B$, of the strip. $A_{0}$ is negative for concave stimuli. The height of the second-order derivative of a Gaussian shape, $h_{2}(x)$, is defined as

$$
h_{2}(x)=B+A_{2} \frac{\sigma^{2}-x^{2}}{\sigma^{2}} e^{-\frac{x^{2}}{2 \sigma^{2}}},
$$

where $A_{2}$ is the amplitude of the shape. Note that the amplitude, just as in Equation 1, equals $h_{2}(0)-B$. The definition of convex and concave is clarified in Figure 1, which shows successively a convex and a concave Gaussian shape, and a convex and a concave second-order derivative of a Gaussian shape. The dimensions of the strip and the dimensions of the Gaussian surfaces are depicted in Figure 2. All strips were $450 \mathrm{~mm}$ long and $30 \mathrm{~mm}$ wide and had a base height, $B$, of $45 \mathrm{~mm}$. In all cases, the surface was practically flat at the ends of the strips.

The stimuli were manufactured on a computer-controlled milling machine, which had an accuracy of $1 \mu \mathrm{m}$. All strips consisted of polyurethane foam filled with artificial resin. The flat reference stimuli had the same proportions and were made of the same materials as the curved ones. They were manufactured in the same batch as the curved stimuli. To ensure that curved and reference stimuli were free of irregularities, they were measured prior to use in a 3-D scanning machine, which also had an accuracy of $1 \mu \mathrm{m}$. The difference between the surface and the desired profile was rarely more than a few micrometers.

We chose two values of the spatial width of the stimuli: narrow ( $\sigma$ equal to $7.5 \mathrm{~mm}$ ), and broad ( $\sigma$ equal to $60 \mathrm{~mm}$ ). These values lie far enough apart for us to measure the influence of spatial width on the thresholds. The 450-mm length of the strips enabled the participants to conveniently scan surfaces with each value of $\sigma$. The choice of amplitudes of the stimuli was made on the basis of pilot experiments. Eight strips of different amplitude were manufactured for each shape and $\sigma$. The amplitudes of the shapes were chosen on an exponential scale. Each surface differed by a factor 1.45 in amplitude from the next one. Of course, the other properties, such as slope and curvature, covaried with amplitude. The ratio between the lowest amplitude and the highest one in a series was about 14. Thus, each series of strips covered a wide range of amplitudes and slopes (see Table 1).

Experimental setup. The participants sat behind a table. A curtain prevented them from seeing the stimuli. The experimenter placed the stimuli on the table, and the participants scanned them with the index and middle fingers of the dominant hand. They had to move these fingers four times over the stimulus surface.

Procedure. A two-alternative forced-choice (2AFC) paradigm was used to find out whether the participants could distinguish a curved stimulus from a flat one. We refer to the thresholds resulting from this procedure as detection thresholds. A flat reference strip and one of the four shapes were presented successively at the same position in random order. Thresholds for different shapes were measured in separate sessions. The participants were told which shape they had to judge from flat during the session. They were asked, "Is the first or the second strip more curved?" The participants scored the answers themselves. Eight different pairs of stimuli were used for each measurement of a threshold. Each one of these pairs was presented 10 times in random order. On average, the participants needed 45 min to complete a session of 80 trials. No feedback was given during the experiment.

Analysis. The percentage of correct answers in the 2AFC task was plotted versus the logarithm of slope (see Figure 3). These

Table 1

Ranges of Absolute Values of Amplitudes (A) and Slopes $(S)$ of the Stimuli

\begin{tabular}{cccc}
\hline$\sigma(\mathrm{mm})$ & Derivative & $A(\mu \mathrm{m})$ & $S$ (min of arc) \\
\hline 7.5 & zeroth & $15-200$ & $4-56$ \\
& second & $7-95$ & $4.3-60$ \\
60 & zeroth & $290-4,000$ & $10-139$ \\
& second & $140-1,900$ & $11-150$ \\
\hline
\end{tabular}




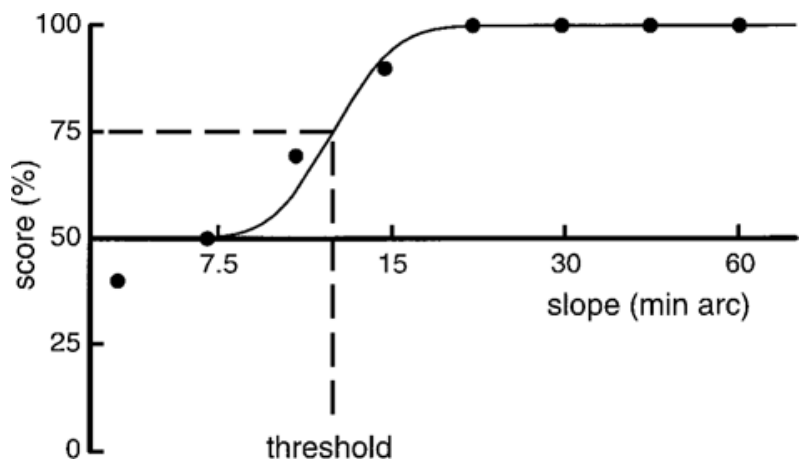

Figure 3. An example of data and the psychometric function that were fitted to these data is shown. The participant's judgment score was plotted versus the maximum slopes of the Gaussian stimulus. The participant discriminated the steepest stimulus easily from a flat surface, which resulted in a score of $100 \%$. The flatter the stimuli, the more the scores were distributed around $50 \%$. The discrimination threshold is defined as the $75 \%$ point on the fitted psychometric curve.

points are equally distributed on the abscissa because the slopes of the stimuli were chosen on an exponential scale. The threshold was defined as the $75 \%$ correct point of the psychometric function $f(z)$,

$$
f(z)=75+25 \operatorname{erf}\left(\frac{z-z_{0}}{\sqrt{2 s}}\right),
$$

where erf is the cumulative Gaussian distribution (see Gautschi, 1964), $z$ the maximum slope of the stimuli, $z_{0}$ the detection threshold, and $\sigma$ the width of the psychometric function. This $s$ is the distance between $66 \%$ and $84 \%$ on the psychometric function. It gives an indication about the accuracy of the determination of the threshold. The function $f(z)$ is fitted to the data by means of the Levenberg-Marquardt method. Independently, both Levenberg (1944) and Marquardt (1963) developed this fast and robust algorithm for the least squares estimation of nonlinear parameters.

It will be advantageous to present the data in a way that allows direct comparison with the findings of our earlier study (Louw et al., 2000). In that study, the main finding was the clear dependence of detection threshold amplitude on $\sigma$ with a power law function:

$$
A_{\text {threshold }} \alpha C_{0} \sigma^{1.3} \text {. }
$$

This can be rewritten as

$$
\frac{A_{\text {threshold }}}{\sigma^{1.3}} \alpha C_{0} .
$$

As slope is proportional to $A / \sigma$, Equation 5 is equivalent to

$$
\frac{S_{\text {threshold }}}{\sigma^{0.3}} \alpha C_{1},
$$

where the $C_{i}$ 's are constants. Here, $A_{\text {threshold }}$ stands for the amplitude, and $S_{\text {threshold }}$ stands for the slope that belongs to the threshold. For convenience, we chose the measure $S_{\text {threshold }} / \sigma^{0.3}$ out of the three equivalent equations as the effective stimulus in that study.

The measure above was devised to compare several possible mechanisms in a simple statistical test. Testing is simple because we only have to check which measure was proportional to a constant. We performed the following statistical analysis in all three experiments in the present study. First, we investigated whether amplitude, slope, slope $/ \sigma^{0.3}$, or maximum local curvature is independent of $\sigma$. Specifically, we tested whether there is a statistical difference between two groups of thresholds, measured for different $\sigma \mathrm{s}$. We tried to find out which measure is proportional to a constant. That measure then stands for the effective haptic stimulus for detection or discrimination thresholds. The measures amplitude, slope, and curva- ture were chosen because these are clear mathematical properties of the stimulus. We chose $S_{\text {threshold }} / \sigma^{0.3}$ because it is the measure reported in haptic literature, as described in the Introduction.

\section{Results}

Figure 4 shows detection threshold slopes in units of minutes of arc for the four different shapes. Light and dark gray bars denote convex and concave stimuli, respectively. On visual inspection, there is no clear-cut dependence of threshold slopes on the four shapes. This is confirmed by a two-way analysis of variance (ANOVA) without interaction with shape and $\sigma$ as parameters. A significant dependence of threshold amplitude and curvature on shape $[F(3,31) \geq 6.1, p \leq .01]$ was found, so neither measure describes the data appropriately. Threshold slopes, on the other hand, showed no significant dependence in this analysis $[F(3,31)=0.93, p=.43]$. This means that the threshold slope gives the best description of the data. Results of the ANOVA are summarized in Table 2. Note that the measures slope and slope $/ \sigma^{0.3}$ yield the same result, because shape and $\sigma$ are not related.

In the present experiments, the ratio between $\sigma$ equal to 7.5 and $60 \mathrm{~mm}$ was 8 . So, when the thresholds have the same dependence on $\sigma$ as thresholds found in our earlier research (Louw et al., 2000), the ratio of threshold slopes must be $8^{0.3}$, which is about 2 . Figure 4 shows that this is indeed the case. As explained in the Analysis section, the measure that is independent of $\sigma$ is the effective stimulus. A one-way ANOVA for each participant with $\sigma$ as parameter showed significant dependencies of amplitude, slope, and curvature on $\sigma[F(1,7) \geq 8.4, p \leq .05]$. No significant dependence of slope $/ \sigma^{0.3}$ on $\sigma$ was found $[F(1,7) \leq$ $1.0, p \geq .05]$, except in the case of J.S. None of the four measures describes the data of J.S. well, because the data lie between a dependence on slope $/ \sigma^{0.3}$ and a dependence on curvature.

\section{Discussion}

In the analysis of our data, the maximum slope of a stimulus proved to be a good first approximation of the effective stimulus for the detection of different shapes. So we chose the threshold slope as a measure to express our data in Figure 4. If the thresholds were expressed as an amplitude, for instance, then the invariance of thresholds for different shapes would vanish. Then, the threshold amplitudes for Gaussian shapes are about twice as high as the thresholds of second-order Gaussian derivatives. This is because a zeroth-order Gaussian derivative with the same slope as a second-order Gaussian derivative has an amplitude that is about two times as high. The independence of threshold slopes on shape corresponds neatly with the literature. Goodwin et al. (1991) and Louw et al. (2000) also found that thresholds for convex and concave shapes do not differ significantly.

\section{EXPERIMENT 2}

In Experiment 1, we established that detection thresholds are independent of the kinds of shapes we used and de- 

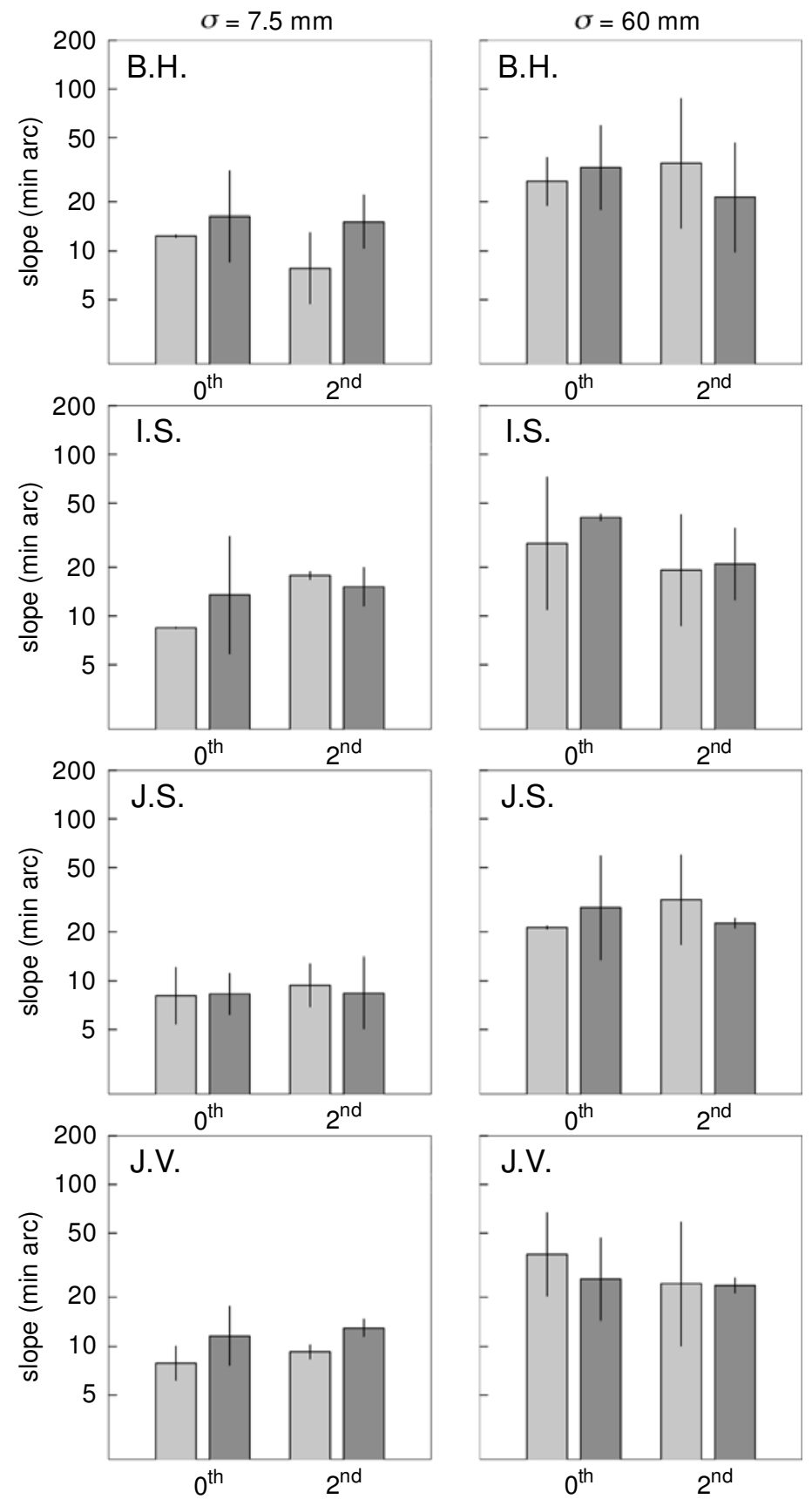

Figure 4. Results for the 4 participants (left upper corner) in Experiment 1 . The detection threshold slopes are plotted in units of minutes of arc against condition. On the left in each panel are the zeroth-order Gaussian derivatives; on the right are the second-order Gaussian derivatives. Light gray bars denote convex stimuli; dark gray bars denote concave ones. Vertical lines indicate the width of the psychometric fit.

pend on $\sigma$ in accordance with haptic literature. The next question is whether these dependencies are a special property of comparing curved stimuli with flat references. To test this, we measured detection thresholds and discrimination thresholds. Of course, detection thresholds were already determined in Experiment 1; however, in Experiment 2, we wanted to compare the two thresholds directly, so the thresholds had to be measured within the same group of 
participants. The necessity for direct comparison within participants stems from the fact that participants have different thresholds.

Detection thresholds were determined for convex shapes only, because neither Experiment 1 values nor literature values of haptic detection thresholds show any differences between thresholds of convex and concave shapes. In the case of detection thresholds, the participants knew that one of the stimuli was flat. In the measurement of discrimination thresholds, the participants were offered a convex stimulus and its concave counterpart. In Experiment 2, the participants had to judge which stimulus was convex.

\section{Method}

Participants. Four students participated: 1 female student (C.V.) and 3 male students (K.O., S.C., and R.H.). They were ignorant of the purpose of the experiment, and they were paid for their efforts. Coren's test (1993) showed that all participants were strongly righthanded.

Stimuli. The experiment was performed with the same four sets of stimuli used in Experiment 1. Detection thresholds were measured for the convex zeroth- and second-order derivatives of a Gaussian shape. Discrimination thresholds were determined for the comparison of convex and concave zeroth-order Gaussian derivatives and for the comparison of convex and concave second-order Gaussian derivatives (see Conditions 1 and 2 in Figure 5). The two detection and two discrimination thresholds were determined for both values of $\sigma$; therefore, eight thresholds were measured for each participant.

Experimental setup and Procedure. Detection thresholds were measured as described in the Procedure section of Experiment 1. Discrimination thresholds were measured as follows. During each series, drawings of the convex shape and the concave shape were shown visually, and one of these shapes was marked. The participants were asked, "Which one of the two strips has the marked shape?" For each determination of a discrimination threshold, we used six pairs of convex and concave stimuli. Apart from an opposite sign, the stimuli within each pair had the same properties. All six pairs were presented to the participants 12 times in random order. The answers were scored by the participants themselves. On average, they needed $40 \mathrm{~min}$ to complete one series, consisting of seventy-two 2AFC choices.

Analysis. The raw data for detection thresholds were analyzed in the same way as were the data in Experiment 1. In the discrimination task, the stimuli that were compared with each other had a different shape; they were convex or concave, but the absolute values of their maximum slopes were the same. The difference between the convex and concave stimuli was taken as the measure for the dis-
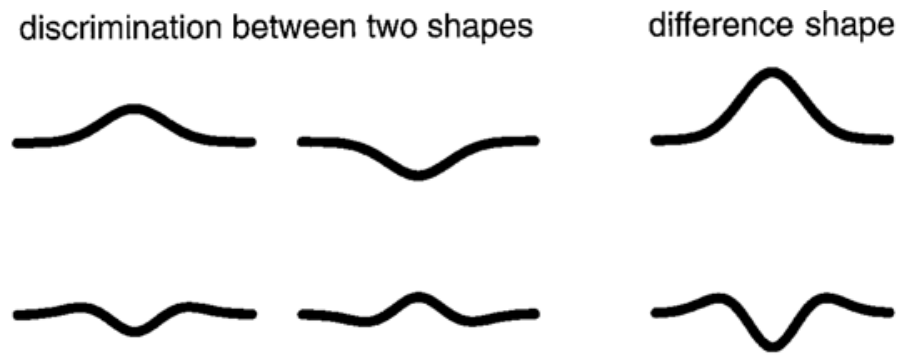

3
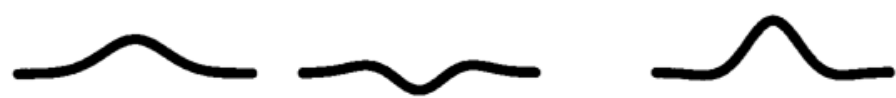

4
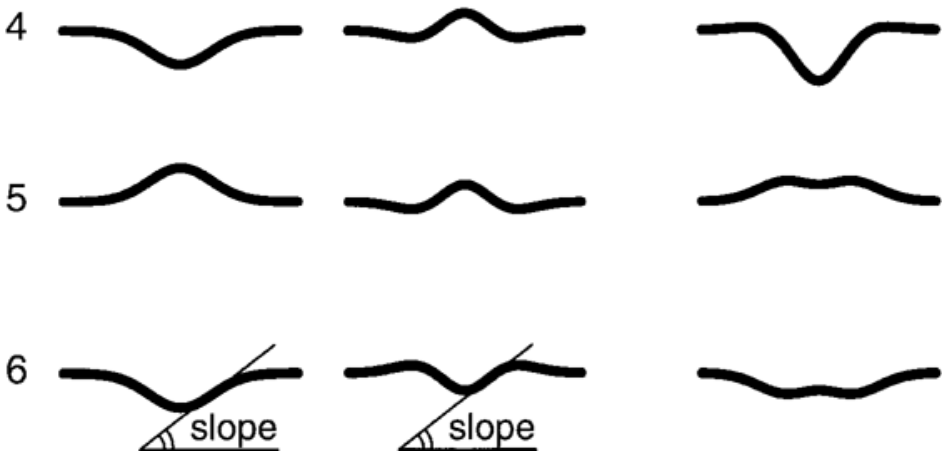

Figure 5. Schematic drawing of conditions (enumerated) for the determination of discrimination thresholds. All six possible combinations of the four shapes (left and middle column) are shown. In the right column, the shapes are subtracted from each other and displayed as a difference shape. The absolute values of the maximum slopes within each pair of stimuli are equal, an example of which is given in Condition 6. 
Table 2

Results of Analyses of Variance

\begin{tabular}{|c|c|c|c|c|c|}
\hline Variable & Participant & Amplitude & Slope & Slope $/ \sigma^{0.3}$ & Curvature \\
\hline \multicolumn{6}{|c|}{ Experiment 1} \\
\hline Shape & All & $*$ & & & * \\
\hline \multirow[t]{4}{*}{$\sigma$} & B.H. & $*$ & $*$ & & $*$ \\
\hline & I.S. & $*$ & $*$ & & $*$ \\
\hline & J.S. & $*$ & $*$ & $*$ & $*$ \\
\hline & J.V. & $*$ & $*$ & & $*$ \\
\hline \multicolumn{6}{|c|}{ Experiment 2} \\
\hline Shape & All & $*$ & & & $*$ \\
\hline$\sigma$ & All & * & $*$ & & $*$ \\
\hline \multicolumn{6}{|c|}{ Experiment 3} \\
\hline Condition & All & & & & \\
\hline$\sigma$ & F.G. & $*$ & & & $*$ \\
\hline & M.K. & $*$ & $*$ & & $*$ \\
\hline & C.L. & $*$ & $*$ & & $*$ \\
\hline & B.O. & $*$ & & & $*$ \\
\hline
\end{tabular}

*Indicates a significant difference ( $p \leq .05$ ) between $\sigma$ equal to $7.5 \mathrm{~mm}$ and $\sigma$ equal to $60 \mathrm{~mm}$.

crimination threshold. The stimuli in the present task are shown schematically as Conditions 1 and 2 in Figure 5. The difference between the shapes in both conditions is shown in the third column of Figure 5.

\section{Results}

Threshold slopes have been plotted against condition in Figure 6 . The conditions are detection and discrimination thresholds. Light gray bars denote zeroth-order Gaussian derivatives; dark gray bars denote second-order Gaussian derivatives. For one data set, K.O. with $\sigma$ equal to $7.5 \mathrm{~mm}$, the fitting procedure did not converge. Figure 6 clearly shows that discrimination thresholds are higher than detection thresholds for almost every participant, shape, and $\sigma$. Overall, discrimination thresholds were about three times as high as detection thresholds. More precisely, a paired $t$-interval test shows that the difference between detection and discrimination thresholds was between 2.3 and 4.4 (95\% confidence). Furthermore, threshold slopes of zeroth- and second-order Gaussian derivatives were not significantly different from each other. A multivariate analysis of variance (MANOVA) was performed with participant, shape, condition, and $\sigma$ as parameters. No significant difference $[F(1,30)=0.06, p=$ $.80]$ could be found between threshold slopes of zerothand second-order Gaussian derivatives in this analysis. These results are shown in Table 2 in the row "Shape" of Experiment 2.

We were interested in the threshold values as a function of stimulus width, $\sigma$. We tested which measure of threshold did not depend significantly on $\sigma$, as described in the Analysis section of Experiment 1. The same MANOVA as described above was used. It showed that the threshold amplitude, the slope, and the curvature all depended significantly on $\sigma[F(1,30) \geq 9.4, p \leq .05]$. The measure threshold slope $/ \sigma^{0.3}$ did not depend significantly on $\sigma[F(1,30)=$ $1.1, p=.30]$. These results were valid for both detection and discrimination thresholds. Table 2 summarizes the results of the ANOVA. The outcome of the statistical analysis was equivalent to the statement that the threshold slope is proportional to $\sigma^{0.3}$.

\section{Discussion}

Discrimination thresholds were about three times as high as detection thresholds. This is well above the factor of 2 that we expected to find. Evidently, each individual stimulus has to be well above detection threshold before participants can tell whether it is convex or concave. It is quite probable that, because the Gaussian shapes are located mainly in the middle of the stimuli, the flat ends of the stimuli provide a robust flat reference for detection. This line of reasoning accounts for a factor of at least 2 between detection thresholds and discrimination thresholds. The fact that a factor higher than 2 has been found can be grasped from the idea that, after detection, participants have to gather more information before they know whether a shape is convex or concave.

Statistical analysis showed that a threshold slope proportional to $\sigma^{0.3}$ gives a good description of the data. Although the absolute values of detection and discrimination thresholds differ substantially, discrimination thresholds do not depend on shape. Discrimination thresholds also have the same dependence on $\sigma$ as detection thresholds found in Experiment 1 and in Louw et al. (2000).

\section{EXPERIMENT 3}

In Experiment 2, we concluded that, although the absolute value of discrimination thresholds was higher than that of detection thresholds, both thresholds showed the same dependence on $\sigma$. In Experiment 3, we tested whether the thresholds for discrimination of six pairs of stimuli behave accordingly. The influence of spatial dimensions and shape on discrimination thresholds was investigated. In order to measure these dependencies, we asked the participants to discriminate different shapes. The set of discrimination threshold conditions was extended to all six possible combinations of the same four shapes used in Experiments 1 and 2. We compared not only convex and concave stimuli but also zeroth- and second-order Gaussian derivatives. So measures had to be taken to prevent the participants from judging the difference between stimuli on the basis of a different slope or, loosely speaking, the "ease" with which each stimulus can be detected. In Experiment 1 , we showed that stimuli with the same maximum slope but different amplitude and shape were equally well discriminated from flat. By creating pairs of stimuli with the same maximum slope, we prevented the participants from recognizing shapes as a result of one of the shapes being more distinct than the other.

\section{Method}

Participants. Four people participated in this experiment: 2 female students (F.G. and C.L.) and 2 male students (M.K. and B.O.). The participants were ignorant of the purpose of the experiment, and 

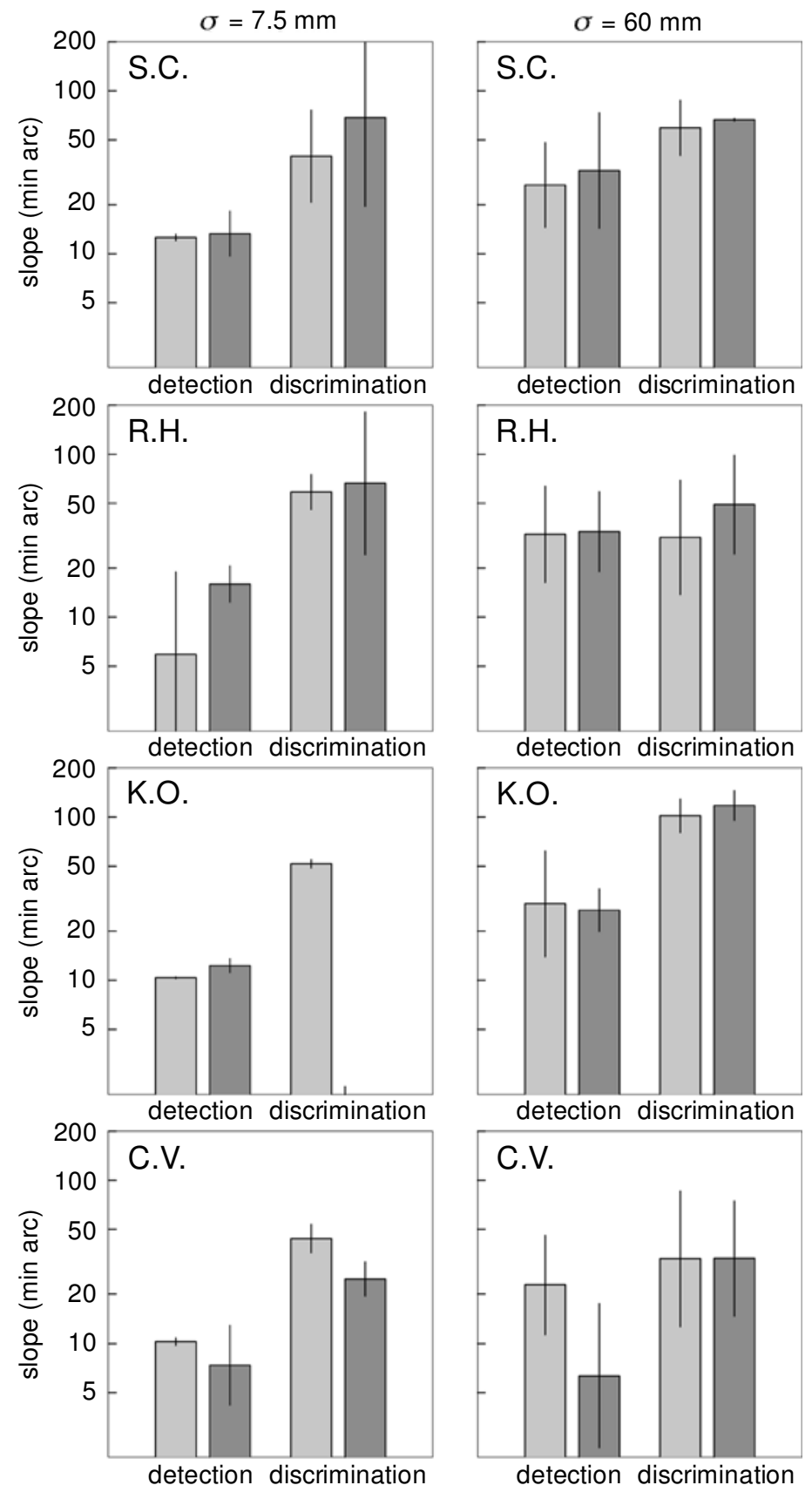

Figure 6. Results for Experiment 2. The detection and discrimination threshold slopes are plotted in units of minutes of arc. Light and dark gray bars denote zeroth- and second-order Gaussian derivatives, respectively. The participant is indicated in the top left corner; $\sigma$ is indicated at the top of the columns. Vertical lines indicate the width of the psychometric fit.

they were paid for their efforts. Coren's test (1993) showed that all participants were strongly right-handed.

Stimuli. The experiment was performed with the same four sets of stimuli used in Experiments 1 and 2. Each of the four shapes was compared with the three others. This yielded six pairs of stimuli, enumerated Conditions 1-6, which are schematically depicted in the left and middle column of Figure 5. The stimuli within a pair have the same maximum slope and a different shape. Conditions 1 and 2, comparing convex and concave zeroth- and second-order Gaussian derivatives, corresponded to the conditions of discrimination thresholds measured in Experiment 2. In the other conditions, the two stimuli differed not only in sign but also in other aspects, such as 
amplitude and the number of local extrema. The absolute values of the maximum slopes within each pair of stimuli were equal. This applied for all conditions (an example is given in Figure 5, Condition 6). We used six pairs of stimuli to determine each threshold. All six pairs were presented to the participants 12 times in random order. Each participant performed 12 series of 2AFC tasks (i.e., 6 conditions $\times 2 \sigma \mathrm{s}$ ). The 12 series were presented to the participants in random order.

Experimental setup and Procedure. Discrimination thresholds were measured with the same setup as we used in Experiment 2. During each measurement of a discrimination threshold, a drawing of the two shapes that had to be distinguished was shown visually, and one of these shapes was marked. The participants were asked, "Which of the two stimuli has the marked shape?" The participants scored the answers themselves. On average, they needed $40 \mathrm{~min}$ to complete one series, consisting of seventy-two 2AFC choices.

Analysis. The stimuli of a pair that were compared in this task had different shapes and equal maximum slopes. In Conditions 3-6 this posed a problem: It was not at all clear which property of which stimulus had to be chosen as a measure of the threshold. In order to solve this indistinctness, we used the analysis described briefly in Experiment 2. The height difference between the two shapes was calculated, which resulted in six "difference shapes," (see the third column in Figure 5). A concave Gaussian shape, for example, that was subtracted from a convex one yielded a Gaussian shape again, but with its amplitude doubled. Subtraction of zeroth- and secondorder derivatives of Gaussian shapes yielded quite different shapes. Note that some of the shapes that are Gaussians in Figure 5 resemble Gabor functions. Young (1987) explained in detail the differences between those functions. The measures of the difference shapes were determined uniquely. The analysis yielded a measure of the difference shape that depended on shape and $\sigma$ in the same way as a detection threshold. Four measures of the difference shapes were tested statistically: amplitude, slope, slope $/ \sigma^{0.3}$, and curvature.

\section{Results}

Results for the discrimination of strips with different shapes are plotted in Figure 7. The discrimination threshold slope is plotted against condition. The fitting procedure did not converge for the data set of M.K. in Condition 2 ( $\sigma$ equal to $7.5 \mathrm{~mm}$ ) and for the data set of B.O. in Condition 6 ( $\sigma$ equal to $60 \mathrm{~mm}$ ). The mean over six conditions is indicated with a horizontal line. Thresholds for stimuli with $\sigma$ equal to $7.5 \mathrm{~mm}$ are plotted in the left panels, and thresholds for stimuli with $\sigma$ equal to $60 \mathrm{~mm}$ are plotted in the right panels.

In order to decide which property of the differenceshapes fitted best to our data, we performed a two-way ANOVA without interaction with condition and $\sigma$ as parameters. For each participant, we tested successively whether the amplitude, slope, slope $/ \sigma^{0.3}$, or curvature of the difference shape depended significantly on condition or $\sigma$. The measure that was independent of both $\sigma$ and condition gave the best description of our data. For each participant, 12 thresholds were measured: 6 conditions $X$ $2 \sigma$ s. Condition had 5 degrees of freedom; $\sigma$ had 1 . Results from the ANOVA-namely, significantly different thresholds $(p<.05)$ for different conditions and different $\sigma \mathrm{s}$ - are indicated by asterisks in Table 2 . Note that the column "slope $/ \sigma^{0.3}$ " in the table shows no significant dependencies on either $\sigma$ or condition. This is exactly what we expected. On the basis of $p$ values for conditions alone, we cannot decide which property best describes our data. The $p$ values for $\sigma$ tell us that $\sigma$ had a significant $(p<.05)$ influence on the value of the thresholds in the case of amplitude and curvature. So amplitude and curvature clearly do not fit. The statistical analysis does not exclude the validity of the measure slope for the data of Participants F.G. and B.O. For these participants, the measures slope and slope $/ \sigma^{0.3}$ of the difference shapes describe the data well $(p>.7)$. The data of the other 2 participants (M.K. and C.L.) can only be described with the measure slope $/ \sigma^{0.3}$.

\section{GENERAL DISCUSSION}

When we first measured detection thresholds over the whole range of spatial scales (Louw et al., 2000), we found the power law dependence of thresholds on $\sigma$ : $A_{\text {threshold }} \propto \sigma^{1.3}$, or, equivalently, $S_{\text {threshold }} \propto \sigma^{0.3}$. This dependence might be specific for the kind of stimuli (i.e., Gaussian-shaped surfaces) that we used in that study. Thus, we still did not know whether the dependence holds for other shapes and whether it holds for discrimination thresholds as well. We tried to answer these questions in the three experiments described in this paper. In this series of experiments, we used two sets of Gaussian shapes and two sets of second-order derivatives of Gaussian shapes. Of course, we could go on and on investigating higher and higher derivatives of the Gaussian shape. We will not embark on this course for several reasons. First, these solid shapes will ultimately pass into a microscale textured surface. Also, the relation between thresholds and spatial scale for zeroth- and second-order Gaussians can be extrapolated toward higher order derivatives, given these two shapes can be combined linearly. There are more indications that the dependence of threshold on spatial scale is not specific for the stimuli used in the present study. One piece of evidence is the fact that convex and concave Gaussian shapes yielded the same thresholds. Moreover, thresholds obtained in other studies (e.g., Gordon \& Morison, 1982; Pont et al., 1999), in which circular cylindrical shapes were used as stimuli, were also in accordance with the $S_{\text {threshold }} \propto \sigma^{0.3}$ dependence.

What conclusions can be drawn from the three experiments taken together? In general, there were differences between the threshold values of the various participants. Since cognitive tasks were not involved, we did not expect nor did we find large differences between participants. Davidson (1972) studied haptic curvature judgment and found only small differences between 32 participants. Ignoring the absolute values of the thresholds and focusing instead on dependencies of the thresholds on shape and $\sigma$, we can answer two research questions. First, we wanted to know whether threshold slopes were independent of shape. Experiment 1 demonstrated that no significant differences could be found between the four shapes investigated. The conclusions from Experiment 2 link up with those from the first. No significant difference could be found between discrimination threshold slopes for zeroth- 

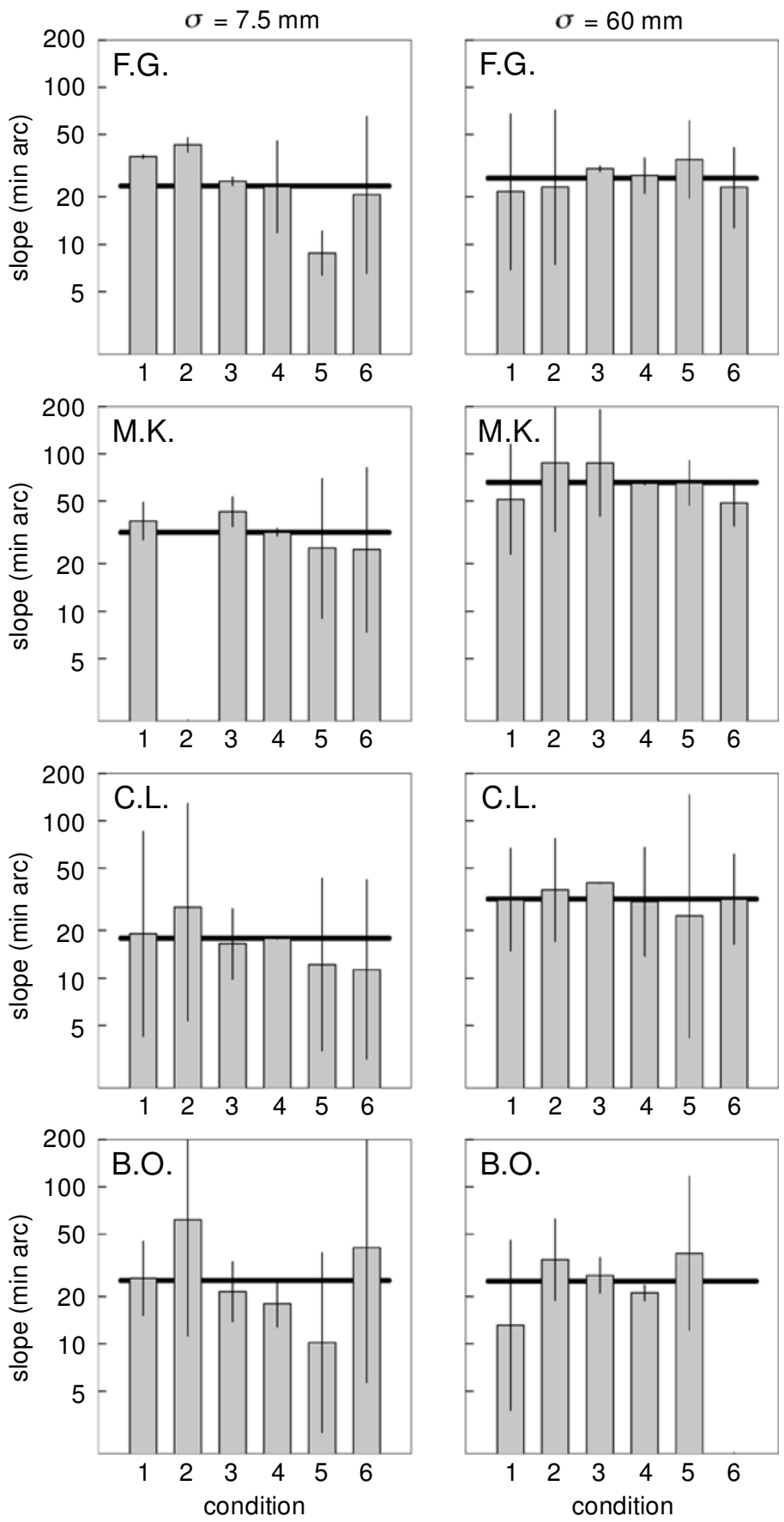

Figure 7. Discrimination threshold slopes in Experiment 3 plotted versus condition, as enumerated in Figure 5. The participant is indicated in top left corner, $\sigma$ is indicated at the top of the columns. Vertical lines indicate the width of the psychometric fit. A horizontal line indicates the mean over conditions.

and second-order derivatives of a Gaussian in Experiment 2. Finally, Experiment 3 showed that, even with six difference shapes, there was no structural dependence of discrimination threshold slope on the shape of the stimuli.
Second, and more specifically, we tested the $S_{\text {threshold }}$ $\propto \sigma^{0.3}$ dependence in all of the present experiments. We tested this statistically by finding the measure, amplitude, slope, slope $/ \sigma^{0.3}$, and curvature of the thresholds that gave 
the best description of our data. A point of theoretical interest here might be the definition of the spatial width of the shapes described. For convenience, we chose the width of a shape to be equal to $\sigma$. Only for the second-order derivative of a Gaussian was there a relatively small difference between $\sigma$ and the distance between the two most extreme slopes. Analyzing all data in the present experiments in terms of $\sigma$ or in terms of the distance between extremes revealed no qualitatively different results between the two measures.

In Experiment 1, the detection threshold slope was proportional to $\sigma^{0.3}$. In Experiment 2, both detection and discrimination thresholds were measured. In spite of differences in the absolute values of the two thresholds, both threshold slopes were in accordance with the $\sigma^{0.3}$ dependence. In Experiment 3, threshold slopes were again proportional to $\sigma^{0.3}$. This stresses the generality of the power law dependence between thresholds and $\sigma$, because the results in Experiment 3 were based on six difference shapes. The dependence of detection and discrimination thresholds on $\sigma$, found in the present experiments, corresponds to the dependence of detection thresholds measured over a broad range of spatial scales in Louw et al. (2000).

A conclusion drawn in Experiment 2 was that, when averaged over participants, shapes, and $\sigma$, the discrimination thresholds were about three times as high as the detection thresholds. To account for this factor of 3 , we concluded that some extra knowledge about the shape of the stimulus must be available after both stimuli have been detected individually. Mountcastle, LaMotte, and Carli (1972) investigated detection and discrimination thresholds of sinusoidal tactile stimuli for humans and monkeys. They correlated psychophysical performance to neural events. Mountcastle et al. concluded that detection thresholds depend on a single discharge in a small number of neurons, whereas discrimination thresholds depend on the pooled response of a large number of neurons. In auditory research, the interval between detection and discrimination is called the atonal region (see Békésy, 1960). Auditory discrimination thresholds are, depending on frequency, 2 to 10 times as high as detection thresholds.

The findings in the three experiments all point in the same direction. Despite differences between participants, a clear pattern is seen when we look at the dependencies of thresholds of individual participants on both shape and $\sigma$ of the stimuli. Given the results in these three experiments, combined with the evidence mentioned in the first part of this General Discussion section, we feel justified in concluding that threshold slope $/ \sigma^{0.3}$ is the effective stimulus for human haptic detection and discrimination of smooth shapes.

A mechanism to explain the specific behavior we found in the present experiments has not yet been pinpointed. Much is known about single mechanoreceptors (see Vallbo, 1995, for a review). By contrast, however, hardly anything is known about the combination of completely different systems, such as skin deformation and cutaneous receptors and proprioception in hand, arm, and shoulder. Even less is known about the kind of processes involved in the integration of signals from distinctly different sources in the somatosensory cortex. The study of Goodwin, Macefield, and Bisley (1997) might shed some light on this issue. They recorded isolated responses from tactile mechanoreceptive afferent fibers in human fingers. Simultaneously with the recording, they successively applied passively five spherical surfaces with different curvatures to the fingertips. In this way, they found a correlation between mechanical deformation, psychophysical reactions, and nerve responses. Goodwin et al. (1997) stated that a population of slowly adapting, Type I afferents (SAI) contains accurate information about the curvature of the stimulus. Research on the responses of cutaneous mechanoreceptors has been central to the work of LaMotte and Srinivasan (1996; Srinivasan \& LaMotte, 1987). LaMotte, Friedman, Lu, Khalsa, and Srinivasan (1998) found clear correlations between millimeter-scale objects applied to the human fingerpads and SA neural responses. They concluded that the spatial distribution of discharge rates, especially those of SA units, encodes the local object shape of an object. This encoding was found to be invariant with changes in object orientation. A different approach to explain the integration between different inputs was taken by Johnson, Hsiao, and Twombly (1995). They attempted to model cortical neuronal responses. They found that neural networks are capable of reproducing the behavior of human observers. These networks, however, do not provide an explanation for human tactile perception. This is because no method of decomposing these neural networks in a meaningful way has yet been found.

A direction that might well lead to the reproduction and simultaneous explanation of human haptic behavior involves the use of a set of differentiating filters. Populations of afferent units with overlapping receptive fields in time and place (see Vallbo, 1995) provide the input for such a mechanism. Cells with receptive fields, sensitive to movement, orientation, and direction, have been found in the hand area of the somatic sensory cortex of primates (see Hyvärinen \& Poranen, 1978). A relatively small number of cells, compared with those in the visual cortex, in the studied population codes for the difference between simple afferent signals by means of postsynaptic lateral inhibition. More complex cortical processing could provide a neural signal indicating the local curvature of a stimulus. In haptic perception, these filters might provide a percept that combines the local slope and the curvature of the shape of an object. The relevant spatial and temporal parameters of this mechanism might be fine-tuned by the adaptation of the human haptic system to its environment.

\section{REFERENCES}

BÉKÉSY, G. VON (1960). Experiments in hearing (E. G. Wever, Trans. and Ed.). New York: McGraw-Hill. Original work published 1958. Coren, S. (1993). The left-hander syndrome. New York: Vintage.

DAvidson, P. W. (1972). Haptic judgments of curvature by blind and sighted humans. Journal of Experimental Psychology, 93, 43-55.

GAUTSCHI, W. (1964). Error function and Fresnel integrals. In M. Abramowitz, \& I. A. Stegun (Eds.), Handbook of mathematical functions (pp. 297-309). New York: Dover.

Goodwin, A. W., John, K. T., \& Marceglia, A. H. (1991). Tactile dis- 
crimination of curvature by humans using only cutaneous information from the fingerpads. Experimental Brain Research, 86, 663-672.

Goodwin, A. W., Macefield, V. G., \& Bisley, J. W. (1997). Encoding of object curvature by tactile afferents from human fingers. Journal of Neurophysiology, 78, 2881-2888.

Gordon, I. E., \& Morison, V. (1982). The haptic perception of curvature. Perception \& Psychophysics, 31, 446-450.

Hyvärinen, J., \& Poranen, A. (1978). Movement-sensitive and direction and orientation-selective cutaneous receptive fields in the hand area of the post-central gyrus in monkeys. Journal of Physiology, 283, 523-537.

Johnson, K. O., Hsiao, S. S., \& Twombly, I. A. (1995). Neural mechanisms of tactile form recognition. In M. S. Gazzaniga (Ed.), The cognitive neurosciences (pp. 253-267). Cambridge, MA: MIT Press.

LaMotte, R. H., Friedman, R. M., Lu, C., Khalsa, P. S., \& SRiniVASAN, M. A. (1998). Raised object on a planar surface stroked across the fingerpad: Responses of cutaneous mechanoreceptors to shape and orientation. Journal of Neurophysiology, 80, 2446-2466.

LaMotte, R. H., \& SRinivasan, M. A. (1996). Neural encoding of shape: Responses of cutaneous mechanoreceptors to a wavy surface stroked across the monkey fingerpad. Journal of Neurophysiology, 67, 3787-3797.

LeVEnberG, K. (1944). A method for the solution of certain non-linear problems in least squares. Quarterly of Applied Mathematics, 2, 164168.

Louw, S., Kappers, A. M. L., \& Koenderink, J. J. (2000). Haptic de- tection thresholds of Gaussian profiles over the whole range of spatial scales. Experimental Brain Research, 132, 369-374.

MARQUARDT, D. W. (1963). An algorithm for least-squares estimation of nonlinear parameters. Journal of the Society for Industrial \& Applied Mathematics, 11, 431-441.

Mountcastle, V. B, LaMotte, R. H., \& Carli, G. (1972). Detection thresholds for stimuli in humans and monkeys: Comparison with threshold events in mechanoreceptive afferent nerve fibers innervating the monkey hand. Journal of Neurophysiology, 35, 122-136.

Pont, S. C., Kappers, A. M. L., \& KoenderinK, J. J. (1999). Similar mechanisms underlie curvature comparison by static and dynamic touch. Perception \& Psychophysics, 61, 874-894.

Srinivasan, M. A., \& LaMotte, R. H. (1987). Tactile discrimination of shape: Responses of slowly and rapidly adapting mechanoreceptive afferents to a step indented into the monkey fingerpad. Journal of Neuroscience, 7, 1682-1697.

VALLBo, $\AA$. B. (1995). Single-afferent neurons and somatic sensation in humans. In M. S. Gazzaniga (Ed.), The cognitive neurosciences (pp. 237-252). Cambridge, MA: MIT Press.

Young, R. A. (1987). The Gaussian derivative model for spatial vision: I. Retinal mechanisms. Spatial Vision, 2, 273-293.

(Manuscript received April 2, 2001; revision accepted for publication December 31, 2001.) 